\title{
The case for intersectionality in ecological psychology
}

\author{
Alexandra Paxton ${ }^{1,2}$, Julia J. C. Blau ${ }^{3}$, Mikayla L. Weston ${ }^{4}$ \\ ${ }^{1}$ Department of Psychological Sciences, University of Connecticut; \\ ${ }^{2}$ Center for the Ecological Study of Perception and Action, University of Connecticut; \\ ${ }^{3}$ Department of Psychological Sciences, Connecticut State University; \\ ${ }^{4}$ Binghamton University, State University of New York
}

Ecological psychology has advanced theory in perception-action-cognition by grounding it within lawful organism-environment relations, allowing us to understand why - not merely how - behavior unfolds. As ecological psychology has grown, it has naturally shifted to examine more interpersonal and social phenomena, embracing early intuitions that the environment is not simply objects and surfaces but also other organisms (Gibson, 1979). This shift has allowed for a fuller explanation of behavior, and we argue for a continuation of that trend: to more fully capture social dynamics by considering how intersectionality impacts social effectivities and affordances. Inspired by work on ecological social meaning (Schmidt, 2007), we here discuss the opportunities, challenges, and research avenues that intersectionality provides ecological psychology.

\section{Intersectionality in Social Affordances and Social Effectivities}

Briefly, the feminist concept of intersectionality holds that different aspects of our identities (e.g., race/ethnicity, gender identity, socioeconomic status) cannot be dissociated from and necessarily interact with one another (Crenshaw, 1991). For feminism and identity theory, intersectionality captures how a person's whole identity constrains their ability to interact with others and engage with their environment. In ecological terms, intersectional identities shape both social effectivities and social affordances. While these abilities and opportunities are largely interpersonal or cultural in nature, the impact of intersectional identities is no less real than physical abilities: The idea that a room full of people affords entering, an empty chair at a crowded table affords sitting, an apartment affords renting, or a job opening affords a paycheck is specific to complex social effectivities based on multiple interacting identities.

Viewed from a purely physical lens, such affordances would be nearly identical for everyone, regardless of social identity. However, this is clearly not the case: People are frequently denied access to physical locations, housing, and economic security based on identity (Ramachandran, 2005). Like physical affordances (Michaels, 2003), certain social affordances do not exist for people without the corresponding social effectivities to realize those affordances. 
Studies in Perception \& Action XV

L. van Dijk \& R. Withagen (Eds.)

Ecological psychology already captures some aspects of intersectionality. For example, the field acknowledges that experiences of and opportunities for action are shaped by different physical bodies and abilities (Franchak \& Adolph, 2013; Kytta, 2004); this can easily be extended to encompass differences in affordances and effectivities for people with versus without physical disabilities. Capturing the impact of other aspects of intersectional identity (e.g., race/ethnicity, sexual orientation) may be somewhat more fraught, but we build our argument on wellestablished work on social affordances and effectivities.

We do not view ourselves as offering claims radically beyond previous social ecological theory. Rather, we propose a straightforward extension prompted by increasing cultural awareness of intersectionality. We directly build on myriad previous claims: Affordances differ by relational identities (e.g., family; Gibson, 1979; Schmidt, 2007); regular but nonspecifying variables help affordance perception and realization (Withagen, 2004); and culture, legal contexts, and histories shape affordances and effectivities (Schmidt, 2007).

The reality is that social effectivities - as an extension of intersectional identity - emerge out of a specific social environment. These effectivities are not representations; they are realities that emerge between people and groups from physical and social properties, sociocultural and legal milieu, and individuals' own histories. Moreover, just as social affordances of an object can be tied to an individual's history with it (e.g., gifts; Schmidt, 2007), social affordances and effectivities tied to intersectional identities may also evolve over time-ultimately being extended beyond a single lifetime to generations of individuals interacting within the same sociocultural environment. Perception and realization of social affordances are constrained by the social effectivities given to us by our sociocultural, temporal, and personal contexts; autopoietically, we find ourselves specified by our sociophysical environment, even as we contribute to the perpetuation of the specifying constraints.

A setback for social affordances is the argument that they do not have the same ontological reality as physical affordances, but we point to previous work on social affordances for a shared defense. Because the affordances are realized through action with the environment, they-like social meaning - exist with no less reality than physical affordances, although they are "relational and higher order than mere physical properties" (p. 143, Schmidt, 2007).

\section{Reciprocity in Social Affordances by Intersectional Identity}

In acknowledging these intersectional social affordances and effectivities, we must grapple with early short-sighted claims that failed to appreciate differences in power and status between humans. Crucially, Gibson claimed that affordances of one organism to another are reciprocal: "What the male affords the female is reciprocal to what the female affords the male; what the infant affords the mother is reciprocal to what the mother affords the infant..." (p. 127, Gibson, 1979). A reasonable interpretation of Gibson's conceptualizations of these "mutual affordances" (p. 127) could situate inter-organism affordances as complementary, with some degree of mutuality and equality. 
Studies in Perception \& Action XV

L. van Dijk \& R. Withagen (Eds.)

However, lived experiences of individuals from groups without institutional power - including women, people of color, the LGBTQ+ community, religious minorities, and other marginalized identities - suggest that social affordances and effectivities are hardly complementary, unless we take the complementarity to encompass outright, unconscious, or institutionalized discrimination. Today, we need not look far to see differences in a person's opportunities for social and physical actions based on their intersectional identity. As just one example, the \#metoo movement has brought into stark relief the differences in what a white man affords a woman of color and what a woman of color affords a white man (Brewer \& Dundes, 2018). To become a unifying theory, ecological psychology must account for underlying causes of documented disparities by intersectional identity (Viruell-Fuentes, Miranda, \& Abdulrahim, 2012); acknowledging differences in social affordances and effectivities would be a valuable start.

Notably, we do not claim that previous work failed to recognize what we might call imbalanced affordances between organisms. For example, Gunns, Johnston, and Hudson (2002) famously studied affordances for attacking another person, and Gibson (1979) pointed to affordances of predators and prey. These capture the imbalance of physical power in a way that we would urge ecological psychology to look to capture differences in social power or status.

\section{Research Proposals for an Intersectional Social Ecological Psychology}

For ecological psychology, intersectionality provides an opportunity for us to situate the entire person in their full context. Rather than reducing a person's existence to merely their physical environment, a social ecological psychology that is sensitive to intersectional identity would capture the person's full history and experience as they move through their physical and social worlds. Embracing intersectionality within social affordances and effectivities does not require cognitivist infrastructure: Instead, we take Schmidt's (2007) perspective that these properties can be directly perceived and attuned to over time by inhabiting a particular physical, social, and cultural environment.

Supporting these claims will require extensive empirical investigation, and we encourage the active social ecological community to critically probe questions around intersectionality in affordances and effectivities. As a first step, we plan to investigate how the affordance of "sit-on-able" might be altered by a chair's context - either situated at a table filled with people who match the participant's intersectional identity or people who might be hostile to it.

As a second potential avenue, we suggest people investigate the intuition that social affordances may be as important as physical ones. Perception is adaptive by specifying valuable information for the organism, affording survival in some cases (Gibson, 1979). From a social perspective, future research could explore the perception by an observer of the intersectional or social identity of an individual interacting within a social and physical environment while obscuring relevant identity cues (e.g., through point-light displays).

Finally, we recognize that our recommendations are neither simple nor straightforward. Just as intersectionality has had to address criticisms about its 
Studies in Perception \& Action XV

L. van Dijk \& R. Withagen (Eds.)

validity or explanatory power (cf. rebuttals by Bredström, 2006; Carbado, 2013), an intersectional social ecological approach will have its own potential concerns. From experiment design to participant selection to statistical analysis, great care will have to be taken to ensure that any effects are driven by underlying realities rather than spurious relations or idiosyncratic responses; particular care should be taken to explore whether identities provide additive versus nonlinear effects. Fully realizing an intersectional social ecological approach will require critical experimental and modelling work by ecological social researchers.

\section{References}

Brewer, S., \& Dundes, L. (2018). Concerned, meet terrified: Intersectional feminism and the Women's March. Women's Studies International Forum, 69, 49-55.

Bredström, A. (2006). Intersectionality: A challenge for feminist HIV/AIDS research? European Journal of Women's Studies, 13(3), 187-192.

Carbado, D. W. (2013). Colorblind intersectionality. Signs, 38(4), 811-845

Crenshaw, K. (1991). Mapping the margins: Intersectionality, identity politics, and violence against women of color. Stanford Law Review, 43(6), 12411299.

Franchak, J. M., \& Adolph, K. E. (2013). Gut estimates: Pregnant women adapt to changing possibilities for squeezing through doorways. Attention, Perception, and Psychophysics, 76, 460-472.

Gibson, J. J. (1979). The ecological approach to visual perception (Classic edition). Boston, MA: Houghton Mifflin.

Gunns, R. E., Johnston, L., \& Hudson, S. M. (2002). Victim selection and kinematics: A point-light investigation of vulnerability to attack. Journal of Nonverbal Behavior, 26(3), 129-158.

Kytta, M. (2004). The extent of children's independent mobility and the number of actualized affordances as criteria for child-friendly environments. Journal of Environmental Psychology, 24(2), 179-198.

Michaels, C. F. (2003). Affordances: Four points of debate. Ecological Psychology, 15(2), 135-148.

Schmidt, R. C. (2007). Scaffolds for social meaning. Ecological Psychology, 19(2), 137-151.

Ramachandran, G. (2005). Intersectionality as "catch 22": Why identity performance demands are neither harmless nor reasonable. Albany Law Review, 69, 299.

Viruell-Fuentes, E. A., Miranda, P. Y., \& Abdulrahim, S. (2012). More than culture: Structural racism, intersectionality theory, and immigrant health. Social Science \& Medicine, 75(12), 2099-2106.

Withagen, R. (2004). The pickup of nonspecifying variables does not entail indirect perception. Ecological Psychology, 16(3), 237-253. 